\title{
Three-dimensional MHD simulations of magnetized molecular cloud fragmentation with turbulence and ion-neutral friction
}

\author{
T. Kudoh ${ }^{1}$ and S. Basu ${ }^{2}$ \\ ${ }^{1}$ National Astronomical Observatory of Japan, Mitaka, Tokyo 181-8588, Japan \\ email: kudoh@th.nao.ac.jp \\ ${ }^{2}$ Department of Physics and Astronomy, University of Western Ontario, \\ London, Ontario N6A 3K7, Canada \\ email: basu@astro.uwo.ca
}

\begin{abstract}
We perform a 3D-MHD simulation of a self-gravitating isothermal gas layer that is initially penetrated by a uniform magnetic field. The strength of the initial magnetic field is such that the cloud is slightly subcritical. In this system, we input random supersonic turbulence initially. Ion-neutral friction is also introduced in the magnetized gas so that the magnetic diffusion allows gas to go across the magnetic field and form self-gravitating cores. We found that self-gravitating cores are formed in the dense region enhanced by the shock waves if ionneutral friction is introduced. The time scale of core formation is on the order of the $10^{6}$ years, which is faster than the usual magnetic diffusion time $\left(10^{7}\right.$ years $)$ estimated from the initial condition. Our result is consistent with the results of 2D-MHD simulations by Li \& Nakamura (2004).
\end{abstract}

Keywords. MHD, turbulence, methods: numerical, stars: formation, ISM: clouds, ISM: magnetic fields

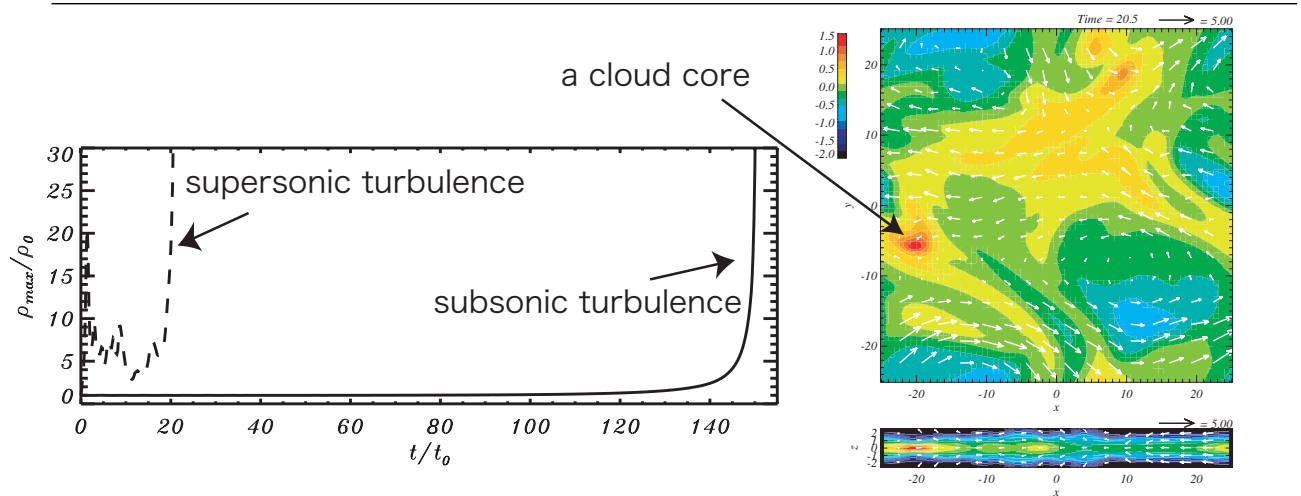

Figure 1. LEFT: Time evolution of maximum density. The dashed line shows the case when the initial perturbation is supersonic (3 times sound velocity). The solid line shows the case when the initial perturbation is subsonic ( 0.1 times sound velocity). The unit of time is $t_{0} \sim 2 \times 10^{5}$ years. RIGHT: Density contour at the final stage for the case of initial supersonic turbulence. White arrows show velocity vectors normalized by sound velocity. Upper panel shows the cross section at $z=0$. Lower panel shows the cross section at $y=-5.9$.

\section{Acknowledgements}

Numerical computations were carried out mainly on VPP5000 at the Center for Computational Astrophysics of the National Astronomical Observatory of Japan. 